\title{
Postoperative tricuspid regurgitation after adult congenital heart surgery is associated with adverse clinical outcomes
}

\author{
Matthew J. Lewis, MD, MPH, Jonathan N. Ginns, MD, ${ }^{\mathrm{a}, \mathrm{b}}$ Siqin Ye, MD,${ }^{\mathrm{c}}$ Paul Chai, MD, \\ Jan M. Quaegebeur, MD, ${ }^{\mathrm{d}}$ Emile Bacha, MD, ${ }^{\mathrm{d}}$ and Marlon S. Rosenbaum, $\mathrm{MD}^{\mathrm{a}}$
}

\begin{abstract}
Objective: Many patients with adult congenital heart disease will require cardiac surgery during their lifetime, and some will have concomitant tricuspid regurgitation. However, the optimal management of significant tricuspid regurgitation at the time of cardiac surgery remains unclear. We assessed the determinants of adverse outcomes in patients with adult congenital heart disease and moderate or greater tricuspid regurgitation undergoing cardiac surgery for non-tricuspid regurgitation-related indications.
\end{abstract}

Methods: All adult patients with congenital heart disease and greater than moderate tricuspid regurgitation who underwent cardiac surgery for non-tricuspid regurgitation-related indications were included in a retrospective study at the Schneeweiss Adult Congenital Heart Center. Cohorts were defined by the type of tricuspid valve intervention at the time of surgery. The primary end point of interest was a composite of death, heart transplantation, and reoperation on the tricuspid valve.

Results: A total of 107 patients met inclusion criteria, and 17 patients $(17 \%)$ reached the primary end point. A total of 68 patients $(64 \%)$ underwent tricuspid valve repair, 8 patients $(7 \%)$ underwent tricuspid valve replacement, and 31 patients $(29 \%)$ did not have a tricuspid valve intervention. By multivariate analysis, moderate or greater postoperative tricuspid regurgitation was associated with a hazard ratio of $6.12(1.84-20.3)$ for the primary end point $(P=.003)$. In addition, failure to perform a tricuspid valve intervention at the time of surgery was associated with an odds ratio of 4.17 (1.26-14.3) for moderate or greater postoperative tricuspid regurgitation $(P=.02)$.

Conclusions: Moderate or greater postoperative tricuspid regurgitation was associated with an increased risk of death, transplant, or reoperation in adult patients with congenital heart disease undergoing cardiac surgery for non-tricuspid regurgitation-related indications. Concomitant tricuspid valve intervention at the time of cardiac surgery should be considered in patients with adult congenital heart disease with moderate or greater preoperative tricuspid regurgitation. ( $\mathrm{J}$ Thorac Cardiovasc Surg 2016;151:460-5)

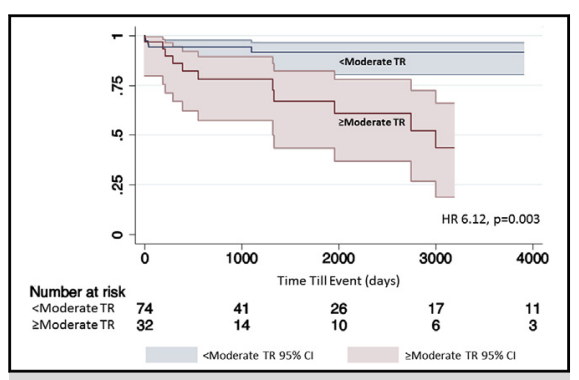

Survivor function for the primary end point by degree of postoperative TR.

\section{Central Message}

$\mathrm{TV}$ intervention at the time of cardiac surgery should be considered in adults with CHD with moderate or greater preoperative TR.

\section{Perspective}

Approximately half of all patients with congenital disease will require repeat cardiac surgery in moderate or greater preoperative TR undergoing cardiac surgery for non-TR-related indications. TV intervention at the time of surgery may decrease the probability of death, TV reoperation, or heart transplant.

See Editorial Commentary page 466.

\footnotetext{
From the a Division of Cardiology, Department of Medicine, Schneeweiss Adult Congenital Heat Center, Columbia University Medical Center, New York, NY; ${ }^{\mathrm{b}}$ Division of Thoracic Imaging, Department of Radiology, Columbia University Medical Center, New York, NY; ${ }^{\mathrm{c} D e p a r t m e n t ~ o f ~ M e d i c i n e, ~ C e n t e r ~ f o r ~ B e h a v i o r a l ~}$ Cardiovascular Health, Columbia University Medical Center, New York, NY; and ${ }^{\mathrm{d}}$ Division of Cardiac, Thoracic and Vascular Surgery, Columbia University Medical Center, New York, NY.

Received for publication April 27, 2015; revisions received Aug 28, 2015; accepted for publication Sept 3, 2015; available ahead of print Oct 26, 2015.

Address for reprints: Matthew J. Lewis, MD, MPH, Herbert Irving Pavilion, 161 Fort Washington Ave, Suite 627, New York, NY 10032 (E-mail: ml3329@cumc. columbia.edu).

$0022-5223 / \$ 36.00$

Copyright (c) 2016 by The American Association for Thoracic Surgery

http://dx.doi.org/10.1016/j.jtcvs.2015.09.028
}

Approximately 1 in 5 adults with congenital heart disease (CHD) will require cardiac surgery during their lifetime, with reoperations accounting for approximately $40 \%$ of these surgeries. ${ }^{1}$ Many of these patients will have concomitant tricuspid regurgitation (TR) before surgery. ${ }^{2}$ The frequent need for cardiac surgery and presence of concomitant tricuspid valve (TV) disease in this population underscore the importance of establishing guidelines addressing the management of TR at the time of cardiac surgery.

The cause of TV disease in adult patients with CHD is variable and includes annular dilation, congenital abnormalities of the TV, iatrogenic leaflet damage from prior 


$$
\begin{aligned}
& \text { Abbreviations and Acronyms } \\
& \begin{aligned}
\mathrm{CHD} & =\text { congenital heart disease } \\
\mathrm{CI} & =\text { confidence interval } \\
\mathrm{PVR} & =\text { pulmonary valve replacement } \\
\mathrm{TR} & =\text { tricuspid regurgitation } \\
\mathrm{TV} & =\text { tricuspid valve }
\end{aligned}
\end{aligned}
$$

surgical procedures, and pacemaker lead placement. ${ }^{3-5}$ Because TR in adults with CHD is often thought to be secondary to other cardiac pathology, intervention on the $\mathrm{TV}$ is often deferred when cardiac surgery is performed for other indications, with the belief that improved ventricular remodeling will lead to a reduction in TR over time. ${ }^{6}$ However, given that TR is associated with increased mortality in the general population ${ }^{7}$ and that TV surgery can be performed with low mortality, ${ }^{8,9}$ it is unclear whether this less aggressive approach to TV repair at the time of cardiac surgery represents an optimal strategy. Furthermore, the risk associated with persistent TR may be even greater in adult patients with $\mathrm{CHD}$, in whom right ventricular dysfunction is more common. ${ }^{10}$ Because of the inherent risk of residual TR and the morbidity conferred by multiple sternotomies, ${ }^{11,12}$ defining a strategy to manage TR at the time of cardiac surgery is crucial in this population. To address this question, we sought to describe the determinants of mortality, heart transplantation, and repeat TV intervention in adult patients with $\mathrm{CHD}$ and moderate or greater TR undergoing cardiac surgery for non-TR-related indications.

\section{MATERIALS AND METHODS \\ Study Design}

We performed a retrospective cohort study of all patients at the Schneeweiss Adult Congenital Heart Center at Columbia University with moderate or greater TR who underwent cardiac surgery for non-TV-related indications between January 1994 and January 2015. The main exposure variable of interest was the type of TV intervention at the time of surgery, defined as no TV intervention, concurrent TV repair, and concurrent TV replacement. The primary end point of interest was a prespecified composite of death, orthotropic heart transplant, and reoperation of the TV. The secondary end point of interest was degree of postoperative TR up to 3 years after surgery. Because we were interested in non-Ebsteinoid TR of the subpulmonic ventricle, patients with congenitally corrected transposition of the great arteries, D-transposition of the great arteries with Mustard or Senning repair, Ebstein's anomaly, and single ventricles were excluded from the study. The Columbia University Medical Center institutional review board approved this study before the onset of study procedures.

\section{Clinical Variables of Interest}

Demographic and clinical data including patient diagnoses and prior surgical procedures were determined through review of electronic and written medical records. Two-dimensional transthoracic echocardiograms performed closest to the time of cardiac surgery were used to define the degree of preoperative TR. Degree of preoperative TR was classified as mild, moderate, moderate-severe, or severe from echocardiographic assessment based on visual assessment performed by 2 cardiologists (JNG and
MSR) with years of expertise in congenital echocardiography. Only patients with moderate or greater TR were included in the study. The degree of postoperative TR was classified as mild or less or moderate or greater using the same methodology and using the last echocardiogram available within a 3-year window from each patient's surgery. Likewise, preoperative echocardiographic right ventricular function was classified as normal or abnormal on the basis of visual echocardiographic assessment by the same readers. Patient-specific data including preoperative heart failure and functional status were ascertained from the patient's clinical visit closest to the time of surgery.

\section{Primary End Point}

The primary end point of interest was prespecified as a composite of death, heart transplant, or reoperation on the TV. Death was determined via review of the medical record and through the Social Security Death Index. Reoperation on the TV was determined via review of the medical records and included any surgeries that were performed for TR. Patients were contacted to assess their current clinical status and to confirm whether cardiac surgery had occurred since the time of last follow-up. In the event that cardiac surgery had been performed, medical records were obtained to adjudicate the primary end point. If a patient was unable to be contacted, follow-up was censored at the time when status of the patient was last known.

\section{Statistics}

Data were expressed as frequency (\%), median (interquartile range), or mean \pm standard deviation as appropriate. Univariate and multivariate testing were performed for the primary end point using a Cox proportional hazard model. Multivariate models for the primary end point were prespecified to contain moderate or greater postoperative TR, age, and concomitant TV intervention based on prior literature. Additional variables reaching $P$ less than .20 in the univariate analysis were subsequently added. Assumption of proportional hazards was verified using a formal significance test based on scaled and unscaled Schoenfeld residuals. Univariate and multivariate testing were performed for the predictors of postoperative TR using logistic regression. Multivariate models for postoperative TR were prespecified to contain age, concomitant TV intervention, and degree of preoperative TR, and any additional variables reaching $P$ less than .20 in the univariate analysis were subsequently added. A Kaplan-Meier survivor function was performed for the primary end point by degree of postoperative TR. Statistical analysis was performed using STATA statistical software (Version 13.1, StataCorp LP, College Station, Tex).

\section{RESULTS}

\section{Patient Characteristics}

Of the 1457 adult patients with CHD who underwent surgery at the Schneeweiss Adult Congenital Heart Center at Columbia University from January 1991 to January 2015, $107(7 \%)$ met inclusion criteria. The median time to the primary end point was 2.96 years (interquartile range, 6.5 years). Patient characteristics are delineated in Table 1. Forty-five $(42 \%)$ of 107 patients were male, and the median age at the time of surgery was 41 years (interquartile range, 14 years). Before cardiac surgery, 55 patients $(51 \%)$ had moderate TR, 21 patients had moderate-severe TR $(19 \%)$, and 31 patients $(30 \%)$ had severe TR. Primary surgeries included 44 pulmonary valve replacements (PVRs) $(41 \%)$, 26 atrial septal defect repairs (24\%), 7 ventricular septal defect repairs (7\%), 6 mitral valve replacements $(6 \%), 6$ Rastelli procedures or conduit replacements $(6 \%)$, and 18 other procedures $(17 \%)$. 
TABLE 1. Patient characteristics

\begin{tabular}{lccc}
\hline \multicolumn{1}{c}{ Variable } & $\begin{array}{c}\text { All } \\
(\mathbf{n}=\mathbf{1 0 7})\end{array}$ & $\begin{array}{c}\text { Event } \\
(\mathbf{n = 1 7 )}\end{array}$ & $\begin{array}{c}\text { No event } \\
(\mathbf{n = 9 0 )}\end{array}$ \\
\hline Gender, male (\%) & $45(42 \%)$ & $8(47 \%)$ & $37(41 \%)$ \\
Age, median, y (IQR) & $41(26)$ & $43(20)$ & $40(22)$ \\
Diagnosis & & & \\
Secundum ASD & $14(13 \%)$ & $1(6 \%)$ & $13(14 \%)$ \\
Sinus venous ASD & $9(8 \%)$ & $1(6 \%)$ & $8(9 \%)$ \\
Partial AV canal/primum ASD & $12(11 \%)$ & $1(6 \%)$ & $11(12 \%)$ \\
VSD & $9(8 \%)$ & $0(0)$ & $9(10 \%)$ \\
Repaired TOF & $30(28 \%)$ & $7(42 \%)$ & $23(25 \%)$ \\
Unrepaired TOF & $2(2 \%)$ & $2(12 \%)$ & $0(0)$ \\
PS & $17(16 \%)$ & $4(24 \%)$ & $13(14 \%)$ \\
DTGA & $4(4 \%)$ & $0(0)$ & $4(4 \%)$ \\
Other & $10(9 \%)$ & $1(6 \%)$ & $9(10 \%)$ \\
RVSP, median (IQR) & $47(31)$ & $51(18)$ & $47(34)$ \\
Preoperative TR & & & \\
Moderate & $55(51 \%)$ & $8(47 \%)$ & $47(52 \%)$ \\
Moderate-severe & $21(19 \%)$ & $2(12 \%)$ & $19(21 \%)$ \\
Severe & $31(30 \%)$ & $7(41 \%)$ & $24(27 \%)$ \\
Tricuspid intervention & & & \\
None & $31(29 \%)$ & $6(35 \%)$ & $24(27 \%)$ \\
Repair & $68(64 \%)$ & $8(47 \%)$ & $61(68 \%)$ \\
Replacement & $8(7 \%)$ & $3(17 \%)$ & $5(5 \%)$ \\
Surgical era & $32(30 \%)$ & $9(53 \%)$ & $23(25 \%)$ \\
1994-2004 & $75(70 \%)$ & $8(47 \%)$ & $67(75 \%)$ \\
2005-2014 & & &
\end{tabular}

$I Q R$, Interquartile range; $A S D$, atrial septal defect; $A V$, atrioventricular; $V S D$, ventricular septal defect; TOF, tetralogy of Fallot; $P S$, pulmonary stenosis; DTGA, D-transposition of the great arteries; $R V S P$, right ventricular systolic pressure; $T R$, tricuspid regurgitation.

At the time of surgery, 68 patients $(64 \%)$ underwent TV repair, 8 patients $(7 \%)$ underwent TV replacement, and 31 patients $(29 \%)$ did not have a TV intervention. Of the 68 patients who underwent TV repair, $13(19 \%)$ underwent an annuloplasty with a tricuspid ring and $55(81 \%)$ underwent a De Vega annuloplasty. One patient who underwent TV repair and a concomitant right atrial Maze procedure had postoperative complete heart block requiring placement of a permanent pacemaker. None of the other patients developed postoperative AV block. In each of the 8 patients who had a TV replacement, a Carpentier-Edwards pericardial xenograft valve (Edwards Lifesciences, Irvine, Calif) was used.

\section{Primary End Point}

Of the 17 patients $(12 \%)$ who met the primary end point, 10 died, 1 underwent heart transplant, and 6 had repeat operations on the TV. There was no significant difference in the number of patients who underwent TV repair or TV replacement who met the primary end point. Specific data for each patient who met the primary end point, including patient diagnosis, age at surgery, type of surgery, type of tricuspid intervention, and patient outcome, are listed in Table 2. By univariate analysis, moderate or greater postoperative TR and age at the time of surgery were significantly associated with the primary end point (Table 3 ). In a multivariate model containing moderate or greater postoperative TR, age at the time of surgery, preoperative congestive heart failure, degree of preoperative TR, and performance of a TV intervention, the presence of moderate or greater postoperative TR remained a significant predictor of the primary end point (hazard ratio, $6.1 ; 95 \%$ confidence interval [CI], 1.84$20.3 ; P=.004)$. Figure 1 displays the survivor function of patients reaching the primary end point by degree of postoperative TR.

TABLE 2. Description of patients meeting the primary end point

\begin{tabular}{|c|c|c|c|c|c|c|c|}
\hline No. & Diagnosis & $\begin{array}{c}\text { Age, } \mathbf{y} \\
\text { (surgery) }\end{array}$ & Surgery & TV intervention & $\begin{array}{c}\text { Year } \\
\text { (surgery) }\end{array}$ & Outcome & $\begin{array}{c}\text { Cause } \\
\text { of death }\end{array}$ \\
\hline 1 & TOF & 58 & TOF repair & None & 1994 & Death & Unknown \\
\hline 2 & TOF & 64 & TOF repair & None & 1997 & Death & Failure \\
\hline 3 & rTOF & 57 & RVOT reconstruction, MVR, Maze & Repaired & 2000 & Death & Failure \\
\hline 4 & rTOF & 38 & PVR & Replaced & 2000 & Death & Failure \\
\hline 5 & rTOF & 63 & PVR, MVR, Maze & Repaired & 2003 & Death & Failure \\
\hline 6 & rTOF & 33 & PVR & Repaired & 2004 & Reoperation & \\
\hline 7 & rTOF & 34 & PVR & None & 2005 & Reoperation & \\
\hline 8 & rTOF & 28 & AVR, PVR & None & 2010 & Reoperation & \\
\hline 9 & rTOF & 56 & PVR, Maze & Repaired & 2010 & Death & Unknown \\
\hline 10 & PS & 40 & PVR & Repaired & 1995 & Reoperation & \\
\hline 11 & PS & 41 & PS resection & None & 2000 & Death & Failure \\
\hline 12 & PS & 48 & PVR & Repaired & 2005 & OHT & \\
\hline 13 & PS & 36 & PVR & Replaced & 2008 & Death & Failure \\
\hline 14 & PAPVR & 64 & Repair, Maze & None & 2004 & Reoperation & \\
\hline 15 & Secundum ASD, hypoplastic RV & 41 & BDG, Maze & Replaced & 2005 & Death & Failure \\
\hline 16 & Secundum ASD & 69 & ASD repair, CABG & Repaired & 2006 & Death & Unknown \\
\hline 17 & AVC & 43 & MV repair & Repaired & 2008 & Reoperation & \\
\hline
\end{tabular}

$T V$, Tricuspid valve; $T O F$, unrepaired tetralogy of Fallot; $r T O F$, repaired tetralogy of Fallot; $R V O T$, right ventricular outflow tract; $M V R$, mitral valve replacement; $P V R$, pulmonary valve replacement; $A V R$, aortic valve replacement; $P S$, pulmonary stenosis; $O H T$, orthotopic heart transplantation; $P A P V R$, partial anomalous pulmonary venous return; $A S D$, atrial septal defect; $R V$, right ventricle, $B D G$, bidirectional Glenn; $C A B G$, coronary artery bypass grafting; $A V C$, atrioventricular canal defect. 
TABLE 3. Univariate and multivariate predictors of the primary end point

\begin{tabular}{|c|c|c|c|c|}
\hline Variable/increment of hazard ratio & $\begin{array}{c}\text { Univariate hazard } \\
\text { ratio }(95 \% \mathbf{C I})\end{array}$ & $P$ value & $\begin{array}{c}\text { Multivariate hazard } \\
\text { ratio }(95 \% \text { CI })\end{array}$ & $P$ value \\
\hline Moderate or greater postoperative TR & $5.3(1.8-15)$ & .002 & $6.12(1.84-20.3)$ & .003 \\
\hline Age at surgery/y & $1.03(0.99-1.07)$ & .05 & $1.02(0.99-1.06)$ & .22 \\
\hline Preoperative CHF & $2.47(0.91-6.69)$ & .08 & $2.60(0.79-8.62)$ & .12 \\
\hline TV intervention & $1.21(0.41-3.57)$ & .72 & $1.7(0.44-6.31)$ & .44 \\
\hline \multicolumn{5}{|l|}{ Degree of preoperative TR } \\
\hline Moderate & Ref & Ref & Ref & Ref \\
\hline Moderate-severe & $0.87(0.18-4.1)$ & .85 & $0.45(0.071-2.94)$ & .41 \\
\hline Severe & $2.4(0.84-7.1)$ & .10 & $1.05(0.29-3.95)$ & .94 \\
\hline Preoperative RV dysfunction & $1.48(0.5-4.43)$ & .47 & & \\
\hline Male gender & $1.1(0.42-2.82)$ & .86 & & \\
\hline $\mathrm{RVSP} / \mathrm{mm} \mathrm{Hg}$ & $0.99(0.96-1.01)$ & .46 & & \\
\hline Tetralogy of Fallot & $1.53(0.56-4.16)$ & .40 & & \\
\hline Pulmonary stenosis & $1.31(0.42-4.04)$ & .63 & & \\
\hline Atrial septal defect & $0.58(0.19-1.79)$ & .35 & & \\
\hline Other diagnosis & $1.19(0.34-4.16)$ & .78 & & \\
\hline \multicolumn{5}{|l|}{ Procedure } \\
\hline ASD repair & $0.40(0.09-1.77)$ & .23 & & \\
\hline PVR & $1.62(0.63-4.22)$ & .32 & & \\
\hline \multicolumn{5}{|l|}{ Surgical era } \\
\hline 2005-2014 & $0.70(0.25-1.96)$ & .50 & & \\
\hline
\end{tabular}

$C I$, Confidence interval; $T R$, tricuspid regurgitation; $C H F$, congestive heart failure; $T V$, tricuspid valve; $R e f$, reference; $R V$, right ventricle; $R V S P$, right ventricular systolic pressure; $A S D$, atrial septal defect; $P V R$, pulmonary valve replacement.

\section{Postoperative Tricuspid Regurgitation}

Thirty-one patients $(32 \%)$ had moderate or greater TR postoperatively. There was a significant difference in the number of patients with moderate or greater TR by year of echocardiogram from time of surgery. By univariate analysis, failure to perform a TV intervention at the time of surgery was associated with 3 times the odds of moderate or greater TR postoperatively $(P=.02)$. In the multivariate analysis, both lack of concomitant TV intervention and severe preoperative TR were significantly associated with the degree of postoperative TR. Specifically, the odds ratio (OR) for developing moderate or greater postoperative

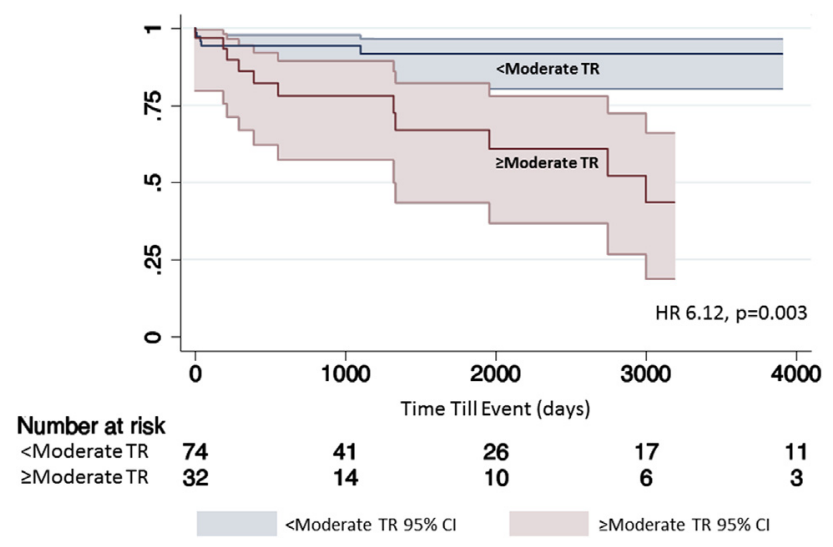

FIGURE 1. Survivor function for the primary end point by degree of postoperative TR. TR, Tricuspid regurgitation; $H R$, hazard ratio; $C I$, confidence interval.
TV was 4.16 (95\% CI, 1.26-14.28; $P=.04)$ for patients who did not have a TV intervention at the time of surgery (Table 4).

Thirty-one patients had severe TR, and 27 patients (87\%) had a concurrent TV intervention. In patients with severe TR, TV intervention was associated with a lower risk of moderate or greater postoperative TR $(P=.026)$. For patients with severe TR, there was no significant difference in the number of patients with moderate or greater postoperative TR by replacement or repair $(P=.071)$. However, preoperative severe TR was associated with 3.6 times the odds of moderate or greater postoperative TR in the multivariate analysis $(95 \% \mathrm{CI}, 1.09-11.9 ; P=.02)$.

\section{DISCUSSION}

The optimal management strategy for TR during adult congenital heart surgery has not been established. In many cases, TR is not surgically addressed in anticipation of improvement in RV dilatation and TV function by correcting an atrial-level shunt or pulmonary regurgitation. ${ }^{13-15}$ However, TR has other causes and is seen in patients with structural abnormalities of the TV, chronic right ventricular hypertension, iatrogenic injury from prior surgeries, pacemaker lead placement, or closure of a ventricular septal defect. ${ }^{16}$ Because of the diverse causes responsible for TR in adults with CHD, it can be difficult to determine whether surgical correction of the primary lesions will also improve a patient's TR. Although recent studies have shown that TV repair can be performed with 
TABLE 4. Univariate and multivariate predictors of postoperative tricuspid regurgitation

\begin{tabular}{|c|c|c|c|c|}
\hline Variable & Univariate OR $(95 \% \mathrm{CI})$ & $P$ value & Multivariate OR $(95 \%$ CI $)$ & $P$ value \\
\hline Age at surgery/y & $1.01(0.98-1.04)$ & .70 & $1.01(0.98-1.04)$ & .40 \\
\hline No TV intervention & $3.03(1.25-7.69)$ & .02 & $4.17(1.26-14.3)$ & .02 \\
\hline \multicolumn{5}{|l|}{ Degree of preoperative TR } \\
\hline Moderate & Ref & Ref & Ref & Ref \\
\hline Moderate-severe & $0.89(0.27-2.89)$ & .85 & $1.94(0.45-8.22)$ & .37 \\
\hline Severe & $2.06(0.80-5.27)$ & .13 & $3.60(1.09-11.9)$ & .04 \\
\hline Preoperative RV dysfunction & $1.96(0.82-4.66)$ & .13 & $1.95(0.76-5.04)$ & .16 \\
\hline Preoperative $\mathrm{CHF}$ & $0.96(0.41-2.22)$ & .94 & & \\
\hline Male gender & $1.29(0.56-2.98)$ & .54 & & \\
\hline $\mathrm{RVSP} / \mathrm{mm} \mathrm{Hg}$ & $1.01(0.99-1.03)$ & .25 & & \\
\hline Tetralogy of Fallot & $0.44(0.15-1.28)$ & .13 & $0.59(0.17-2.01)$ & .40 \\
\hline Pulmonary stenosis & $0.87(0.28-2.68)$ & .81 & & \\
\hline ASD & $1.33(0.55-3.17)$ & .52 & & \\
\hline Other diagnosis & $1.47(0.48-4.48)$ & .49 & & \\
\hline \multicolumn{5}{|l|}{ Procedure } \\
\hline ASD repair (any) & $1.5(0.56-3.9)$ & .43 & & \\
\hline PVR & $0.72(0.30-1.7)$ & .45 & & \\
\hline
\end{tabular}

$O R$, Odds ratio; $C I$, confidence interval; $T V$, tricuspid valve; $T R$, tricuspid regurgitation; $R e f$, reference; $R V$, right ventricle; $C H F$, congestive heart failure; $R V S P$, right ventricular systolic pressure; $A S D$, atrial septal defect; $P V R$, pulmonary valve replacement.

low mortality in this population, ${ }^{8}$ there are little data comparing outcomes of patients who did and did not undergo concomitant TV intervention when surgery is performed primarily for another indication. To answer this question, we excluded patients with Ebstein's anomaly, systemic right ventricles, and single ventricles. In so doing, we found that patients who were left with moderate or greater TR after cardiac surgery were at significantly increased risk of death, heart transplant, or reoperation on the TV. In addition, we found that patients who did not have an intervention on the TV at the time of surgery were at an increased risk of having moderate or more postoperative TR.

Although worsening TR also may be associated with poor outcomes in CHD, the management of TR in patients undergoing procedures for other indications remains variable. Kogon and colleagues ${ }^{6}$ showed that in patients with tetralogy of Fallot and pulmonary stenosis undergoing PVR for pulmonary regurgitation, the degree of TR was not significantly different between patients who did and did not have a TV annuloplasty at the time of surgery. However, this study was limited to a small group of 35 patients that included both pediatric and adult patients. Likewise, Cramer and colleagues ${ }^{17}$ found no significant difference in the degree of postoperative TR in patients who did and did not undergo TV repair at that time of PVR. In contrast, in our cohort of 107 adult patients with CHD undergoing cardiac surgery for non-TR-related indications, we found that patients who did not have a TV intervention had higher odds of having postoperative TR, particularly in patients with severe TR at the time of surgery. Several factors may explain the difference in findings. The median age of our cohort was 12 years greater, possibly allowing for worsening TV annular dilation necessitating repair. Our larger sample size and greater follow-up time may have also provided the power necessary to detect a significant difference. Although underpowering is a possibility, we did not find that any 1 diagnosis was significantly associated with a greater risk of postoperative TR. Furthermore, we found that moderate or greater postoperative TR was the only significant predictor of death, transplant, or repeat operation on the TV. We believe this finding underscores the need to intervene on the TV at the time of cardiac surgery in this population.

These findings may have important clinical implications for the subset of patients who might otherwise undergo percutaneous PVR in the presence of significant TR. In this population, it is unclear whether to pursue a percutaneous strategy or to subject patients to repeat surgery to address both the pulmonary and TV abnormalities. Larger, multicenter studies looking exclusively at this group would be necessary to elucidate the best management strategy; however, our findings suggest that these patients may be at increased risk if left with moderate or greater TR.

TV repair can be performed with low mortality in this population, and several studies have shown improved outcomes when TV surgery is performed before the onset of heart failure in a general cohort. ${ }^{18-20}$ Late repair and reoperation carry substantial increased risks over repair at the time of concomitant cardiac surgery. ${ }^{21}$ This may be especially true in our population of adults with CHD, many of whom have had multiple prior sternotomies. In these patients, chronic moderate or greater TR is likely to result in significant right ventricular remodeling leading to progressive right ventricular dilation and dysfunction. In addition, protracted TR can also result in significant right 
atrial enlargement and predispose patients to atrial tachyarrhythmias. Adult patients with CHD are at increased risk for these complications because many forms of CHD involve right-sided cardiac abnormalities. In our study, all adjudicated deaths were secondary to cardiac failure. Thus, although we are unable to show a direct correlation between death or heart transplant with degree of TR, our findings suggest that such an association may exist. Furthermore, we found that patients with the highest degrees of preoperative TR and those who did not have a TV intervention were at higher risk of having significant postoperative TR. These findings suggest that even in patients who are not symptomatic at the time of surgery, significant TR should be addressed at the time of surgical repair for other lesions.

\section{Study Limitations}

Because this was a retrospective study, patients were not randomized to different treatment groups, and patients undergoing valve replacement and valve repair were aggregated into a single cohort. In addition, a disproportionate number of critically ill patients may have undergone a TV replacement, limiting our ability to show a relationship between the TV intervention and the primary outcome. Furthermore, because this was a retrospective study, we were unable to show direct causation between the TR and the primary end point or TV intervention and the primary end point. As a heterogeneous group of patients, our sample may have been too small to detect differences between different anatomic lesions examined. Nonetheless, to our knowledge, this is the largest study of adult patients with CHD seeking to address this question. Because we limited the follow-up of our patients to 3 years for this study, our ability to draw conclusions on the durability of outcomes over the medium to long-term may be limited. Finally, as a single-center study performed at an institution with expertise in adult CHD, our results may not be generalizable to all centers.

\section{CONCLUSIONS}

Moderate or greater postoperative TR was associated with a significantly increased risk of death, heart transplant, or TV reoperation in adult patients with CHD undergoing cardiac surgery for a non-TR-related indication. In addition, adult patients with CHD with moderate or greater preoperative TR who did not undergo a TV intervention at the time of cardiac surgery had increased odds of having moderate or greater postoperative TR. Although confirmatory prospective studies are required, these findings suggest that, in this population, concomitant $\mathrm{TV}$ intervention at the time of cardiac surgery should be considered in patients with moderate or greater preoperative TR.

\section{Conflict of Interest Statement}

Authors have nothing to disclose with regard to commercial support.

\section{References}

1. Zomer AC, Verheugt CL, Vaartjes I, Uiterwaal CS, Langemeijer MM, Koolbergen DR, et al. Surgery in adults with congenital heart disease. Circulation. 2011;124:2195-201.

2. Said SM, Burkhart HM, Dearani JA. Surgical management of congenital (nonEbstein) tricuspid valve regurgitation. Semin Thorac Cardiovasc Surg Pediatr Card Surg Annu. 2012;15:46-60.

3. Giamberti A, Chessa M, Ballotta A, Varrica A, Agnetti A, Frigiola A, et al. Functional tricuspid valve regurgitation in adults with congenital heart disease: an emerging problem. J Heart Valve Dis. 2011;20:565-70.

4. van Slooten YJ, Freling HG, van Melle JP, Mulder BJ, Jongbloed MR, Ebels $\mathrm{T}$, et al. Long-term tricuspid valve prosthesis-related complications in patients with congenital heart disease. Eur J Cardiothorac Surg. 2014 45:83-9.

5. Ginns J, Ammash N, Bernier PL. The tricuspid valve in adult congenital heart disease. Heart Fail Clin. 2014;10:131-53.

6. Kogon B, Patel M, Leong T, McConnell M, Book W. Management of moderate functional tricuspid valve regurgitation at the time of pulmonary valve replacement: is concomitant tricuspid valve repair necessary? Pediatr Cardiol. 2010;31: 843-8.

7. Nath J, Foster E, Heidenreich PA. Impact of tricuspid regurgitation on long-term survival. J Am Coll Cardiol. 2004:43:405-9.

8. Said SM, Dearani JA, Burkhart HM, Connolly HM, Eidem B, Stensrud PE, et al. Management of tricuspid regurgitation in congenital heart disease: is survival better with valve repair? J Thorac Cardiovasc Surg. 2014;147: 412-9.

9. Holst KA, Dearani JA, Burkhart HM, Connolly HM, Warnes CA, Li Z, et al. Reoperative multivalve surgery in adult congenital heart disease. Ann Thorac Surg. 2013;95:1383-9.

10. Warnes CA. Adult congenital heart disease importance of the right ventricle. $J$ Am Coll Cardiol. 2009;54:1903-10.

11. Kavarana MN, Savage A, O'Connell R, Rubinstein CS, Flynn-Reeves J, Joshi K et al. Composite risk factors predict survival after transplantation for congenital heart disease. J Thorac Cardiovasc Surg. 2013;146:888-93.

12. Holst KA, Dearani JA, Burkhart HM, Connolly HM, Warnes CA, Li Z, et al. Risk factors and early outcomes of multiple reoperations in adults with congenital heart disease. Ann Thorac Surg. 2011;92:122-30.

13. Szymanski P, Lipczynska M, Klisiewicz A, Hoffman P. Clinical settings leading to presystolic tricuspid regurgitation. Echocardiography. 2015;32: 19-27.

14. Toyono M, Krasuski RA, Pettersson GB, Matsumura Y, Yamano T, Shiota T. Persistent tricuspid regurgitation and its predictor in adults after percutaneous and isolated surgical closure of secundum atrial septal defect. Am J Cardiol. 2009; 104:856-61.

15. Toyono M, Fukuda S, Gillinov AM, Pettersson GB, Matsumura Y, Wada N, et al Different determinants of residual tricuspid regurgitation after tricuspid annuloplasty: comparison of atrial septal defect and mitral valve prolapse. J Am Soc Echocardiogr. 2009;22:899-903.

16. Matyal R, Wang A, Mahmood F. Percutaneous ventricular septal defect closure with amplatzer devices resulting in severe tricuspid regurgitation. Catheter Cardiovasc Interv. 2013;82:E817-20.

17. Cramer JW, Ginde S, Hill GD, Cohen SB, Bartz PJ, Tweddell JS, et al. Tricuspid repair at pulmonary valve replacement does not alter outcomes in tetralogy of Fallot. Ann Thorac Surg. 2015;99:899-904.

18. Raikhelkar J, Lin HM, Neckman D, Afonso A, Scurlock C. Isolated tricuspid valve surgery: predictors of adverse outcome and survival. Heart Lung Circ. 2013;22:211-20.

19. Guenther T, Noebauer C, Mazzitelli D, Busch R, Tassani-Prell P, Lange R. Tricuspid valve surgery: a thirty-year assessment of early and late outcome. Eur J Cardiothorac Surg. 2008;34:402-9.

20. Chen SW, Tsai FC, Tsai FC, Chao YK, Huang YK, Chu JJ, et al. Surgical risk and outcome of repair versus replacement for late tricuspid regurgitation in redo operation. Ann Thorac Surg. 2012;93:770-5.

21. Pfannmuller B, Moz M, Misfeld M, Borger MA, Funkat AK, Garbade J, et al Isolated tricuspid valve surgery in patients with previous cardiac surgery. $J$ Thorac Cardiovasc Surg. 2013;146:841-7.

Key Words: congenital heart disease, tricuspid valve repair, tricuspid regurgitation 